\title{
FAR-INFRARED NARROW-BAND PHOTODETECTOR BASED ON MAGNETICALLY TUNABLE CYCLOTRON RESONANCE-ASSISTED TRANSITIONS IN PURE $n$-TYPE InSb
}

\author{
L.H. DMowski ${ }^{a}$, M. Cheremisin ${ }^{b}$, C. SkIERBISZEWSKi ${ }^{a}$ AND W. KNAP ${ }^{b}$ \\ ${ }^{a}$ High Pressure Research Center, Polish Academy of Sciences \\ Sokołowska 29/37, 01-142 Warszawa, Poland \\ ${ }^{b}$ G.E.S. Université Montpellier II, Pl. Eugene Bataillon, 34060 Montpellier, France
}

We present the possibility of using magnetic field to enhance responsivity and to tune spectral range of far-infrared InSb detector (based on photoconductivity effect) beyond its standard range limited to about $30 \mathrm{~cm}^{-1}$. We show that due to cyclotron resonance assisted transitions we can use it as a tunable detector working up to energies about $180 \mathrm{~cm}^{-1}(22 \mathrm{meV})$. We have used such a detector as a spectrometer for measurements of the Landau emission from GaAs emitter.

PACS numbers: 72.40.+w, 78.20.Ls, 78.90.+t

\section{Introduction}

Pure $n$-type InSb has been known to be a high sensitive and fast response far-infrared detector based on photoconductivity effect [1]. It is currently used in the range up to about $30-40 \mathrm{~cm}^{-1}$ due to hot electron effects related to free carrier absorption. Application of magnetic field increases the magnitude of photoresponse by increasing the detector resistance. On the other hand, magnetic field splits the conduction band into Landau levels. The detector absorption and spectral response are then dominated by cyclotron resonance processes, hence being tunable. At $4.2 \mathrm{~K}$ the photoconductive signal at a given magnetic field contains two maxima both of which shift with magnetic field. One of them corresponds to the so-called conduction-electron cyclotron resonance (CCR) $0^{+}-1^{+}$Landau level transition. The second one (the so-called impurity shifted cyclotron resonance (ICR)) corresponds to the transition between impurity states associated with respective Landau levels. We studied a possibility of using magnetic field to enhance and to tune the spectral responsivity of InSb detector, developing the idea presented by Gornik in his works concerning radiative transitions between Landau levels [2]. We show that we can use InSb as a narrow-band and tunable detector up to about $22 \mathrm{meV}\left(180 \mathrm{~cm}^{-1}\right)$ where its efficiency is limited by the reststrahlen (wave number of transversal optical TO-phonon is $1 / \lambda_{\text {TO }}=185 \mathrm{~cm}^{-1}$ ). 


\section{Experiment and results}

We used standard InSb hot electron bolometer type QFI/X produced by the Queen Mary College Instruments with a thickness of $300 \mu \mathrm{m}$ (and free electron concentration of the order of $10^{14} \mathrm{~cm}^{-3}$ at $77 \mathrm{~K}$ ), varnished to a wedged quartz substrate. We measured responsivity spectra of the detector at various fixed values of magnetic field using a Fourier transform spectrometer. The detector was placed in a helium cryostat in the centre of superconducting solenoid generating uniform magnetic field. The detector was mounted on a sample holder coupled to the interferometer with a light pipe. The responsivity spectra were measured with $15 \mu \mathrm{A}$ current bias for detector magnetic field from $0 \mathrm{~T}$ to $1.4 \mathrm{~T}$ with a step $0.1 \mathrm{~T}$ and with $3 \mu \mathrm{A}$ current bias from 1.4 $\mathrm{T}$ to $3.0 \mathrm{~T}$ with a step $0.2 \mathrm{~T}$. Calibration of the spectra was accomplished using incident radiation power spectrum obtained by correctly scaled Ge:Ga bolometer placed in the same position as the detector of interest. Figure 1 shows the shift of the spectral range of photoconductive sensitivity with

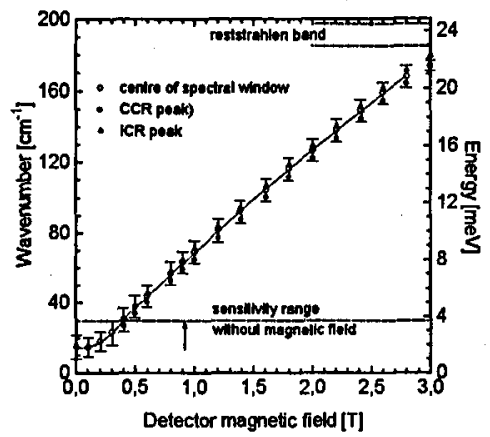

Fig. 1. The shift of spectral range of InSb photodetector with applied magnetic field. "Error bars" stand for half width of photoconductive signal at a given, fixed magnetic field. Solid line represents two polynomials of second order describing the shift of the centre of the spectral width with magnetic field.

magnetic field applied to the detector. "Error bars" marked in the picture stand for half width of photoconductive signal at the fixed magnetic field. ICR and CCR maxima are marked by triangles and circles respectively. The shift of the centre of the spectral width with magnetic field can be well described by a polynomial of the second order with coefficients: $A_{0}=4.1078, A_{1}=68.4495, A_{2}=-3.5259$ for $B>0.4 \mathrm{~T}$ in the cyclotron absorption range and with $A_{0}=14.5766, A_{1}=-7.0314$ and $A_{2}=118.4286$ for $B<0.4 \mathrm{~T}$ in the free carrier absorption range (with energy expressed in $\mathrm{cm}^{-1}$ ). The average detector sensitivity increases with magnetic

- field, reaches a maximum at about $2.5 \mathrm{~T}$ then rapidly falls and vanishes above $3 \mathrm{~T}$ approaching reststrahlen band. At higher magnetic fields the photoconductive effect can be observed again on the high energy side of the reststrahlen band. We have verified this making measurements also at $4 \mathrm{~T}, 5 \mathrm{~T}$ and $6 \mathrm{~T}$. The evolution of the average detector sensitivity with magnetic field (starting from $0.2 \mathrm{~T}$ ), normalised to 1 at the maximum at $2.5 \mathrm{~T}$ can be well described by a polynomial of 


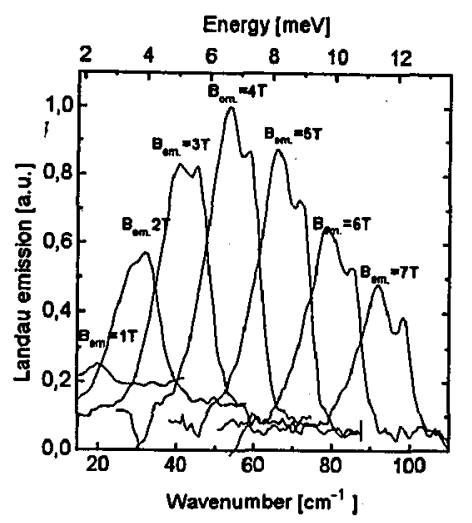

Fig. 2. Relative intensity and position in energy of Landau emission from GaAs (taken by means of InSb spectrometer) for various magnetic fields applied to the emitter.

the eighth order with coefficients: $A_{0}=0.7887, A_{1}=-3.4825, A_{2}=13.5285$, $A_{3}=-28.8027, A_{4}=36.1695, A_{5}=-26.3053, A_{6}=10.7849, A_{7}=-2.3021$, and $A_{8}=0.19835$. Analytical description of the shift of the detector spectral window as well as of the average detector sensitivity with applied magnetic field allowed us to test the detector using it as a spectrometer. Although the spectral resolution of such a spectrometer is relatively low we were able to detect and to analyze far-infrared radiation (FIR) related to the transition between Landau levels in GaAs sample. The experimental setup consisted of two superconducting coils one of which was used to tune the InSb detector while the second to split GaAs conduction band into Landau levels. All elements: FIR emitter and InSb detector were placed in the same cryostat. In order to heat free electrons and thus to obtain inversion of population of Landau levels electric field impulses of the order of $25 \mathrm{~V} / \mathrm{cm}$ were applied to the emitter. Detector magnetic field was swept with a speed of $10^{-2} \mathrm{~T} / \mathrm{s}$. The results showing relative intensity and position in energy of Landau emission from GaAs for various magnetic fields applied to the emitter are presented in Fig. 2.

\section{Conclusions}

In conclusions we can state that standard InSb detector coupled with magnetic field can be a very fruitful tool for studies of FIR radiation from solids. The detector coupled with permanent rare-earth magnet supplying a field of the order of $0.5 \mathrm{~T}$ considerably enhances its responsivity and enlarges its standard spectral range from 30 to $50 \mathrm{~cm}^{-1}$. Detector with a thickness below $100 \mu \mathrm{m}$ (allowing to discriminate in favour to resonant against free carrier absorption) coupled with a fixed or tuned magnetic field can be used as a selective detector. If coupled with a tuned magnetic field (supplied for example by mini superconducting coil), such a detector can be used as a spectrometer in the range $20-170 \mathrm{~cm}^{-1}$. Although its spectral resolution is rather low, in studies of FIR radiation from solids it has some advantages over Fourier transform spectrometer. The latter is usually placed far away from the emitter and kept at room temperature, so the noise due to $300 \mathrm{~K}$ 
radiation can obscure the investigated signal. The spectral resolution (limited by the width of CCR line) can be improved if we could use two-dimensional InSb structure with much higher mobility.

\section{Acknowledgments}

We acknowledge technical support by Jose Ortega. One of us (M.Ch.) was partially supported by INTAS fellowship program.

\section{References}

[1] E.H. Putley, in: Semiconductors and Semimetals, Eds. R.K. Willardson, A.C. Beer, Vol. 1, Academic Press, New York 1966, p. 289.

[2] E. Gornik, in: Lecture Notes in Physics, Ed. G. Landwehr, Vol. 177, Springer-Verlag, Berlin 1983, p. 248. 